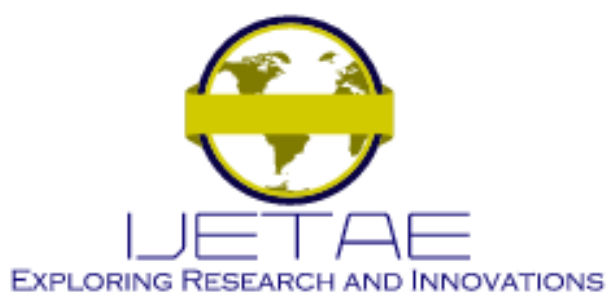

International Journal of Emerging Technology and Advanced Engineering

Website: www.ijetae.com (E-ISSN 2250-2459, Scopus Indexed, ISO 9001:2008 Certified Journal, Volume 11, Issue 06, June 2021)

Manuscript Received: 14 April 2021, Received in Revised form: 25 May 2021, Accepted: 05 June 2021

DOI: 10.46338/ijetae0621_02

\title{
Promotion Scenario Based Sales Prediction on E-Retail Groceries Using Data Mining
}

\author{
Avicienna Ulhaq Muqodas ${ }^{1}$, Gede Putra Kusuma ${ }^{2}$ \\ Computer Science Department, BINUS Graduate Program - Master of Computer Science, Bina Nusantara University, Jakarta, \\ 11480, Indonesia
}

\begin{abstract}
Mostly in many business cases, sales prediction plays an important role. Production planning is a good example. One aspect which affecting sales forecasting is promotion schedule. Since using promotion is commonly done nowadays, especially in internet business, it is hardly seen a day without promotion in Indonesian e-commerce. Thus, this study discusses about forecasting future sales based on promotion scenario data with main objective is to discover the best machine learning algorithm and model to forecast future sales. Promotion mechanism which employed in this study are price cut, buy 1-get 1, and product bundling. We use 577 data from January 2018 to July 2019 as dataset. We compare kNN, GLM, and SVR as the model predictor to forecast number of transactions in a day. From the experiment k-NN yielded the highest performance ability with squared correlation of $\mathbf{0 . 9 3 8}$. the worst model predictor for this case is GLM with squared correlation of 0.507 . We also determine the best parameter input for each parameter using grid optimization method. We discover 2 is the best $k$ value of $k$ NN and Manhattan distance is the best distance calculation for this case.
\end{abstract}

Keywords - Data Mining, Nearest Neighbor, Online Retail, Promotion Based, Sales Prediction

\section{INTRODUCTION}

Mostly in many business cases, sales prediction plays an important role. Production planning is a good example. In digital business, estimating sales in the future also have an important role. Resource planning is one of them. Usually, the increment of sales, parallel with resource usage such as server, network, or database. Misprediction of future sales, bring one or another loss. First, if the resources have a larger capacity than predicted sales, they will be experienced over-resources, resulting decremental of resource utilization.
Second, if the resources have a smaller capacity than predicted sales, they will be experienced over demand, which results in system failure. In this event, loss occurs not only in tangible form, such as sales loss per downtime, but also in intangible form such as image brand or customer experience. To this end, have a good prediction of future sales is an imperative need for every company. Table 1 from experiment done by [1] explain this scenario by comparing server size, with their cost, with addition of request per second and cost per 100 request per second, which illustrate the larger size of the server is costlier in a certain number of requests per second. The server with the largest size, although have the highest cost per hour, but could serve 320 requests per second which produces a lower cost of 100 requests per second compared with the smallest server size, which only serves 92 requests per second. So, knowing have many requests to serve is imperative to maintain the cost of infrastructure. Moreover, [2] explain in detail how sales forecasting impact whole organization in terms of forecasting scope, either in shortterm or long-term.

Many business cases already used as a subject in this area of study, for example, sales forecasting in tourism sector already studied by [3] which use linear and nonlinear to forecast tourism demand in Taiwan. Media and entertainment also have been studied [4]. They studied about product sales forecasting using online search data, they concluded that online search data offer predictive power in media and entertainment business. Studies about sales prediction has been done in variate business cases, and variate approach. Generally, prediction techniques can be divided into two major categories: non-algorithmic and algorithmic, or statistical model and computational intelligence [5]. 


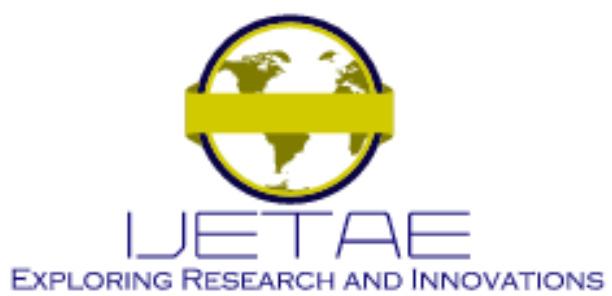

International Journal of Emerging Technology and Advanced Engineering

Website: www.ijetae.com (E-ISSN 2250-2459, Scopus Indexed, ISO 9001:2008 Certified Journal, Volume 11, Issue 06, June 2021)

TABLE 1

NUMBER OF REQUEST VS COST SCENARIO

\begin{tabular}{|l|c|c|c|c|}
\hline Server Size & Cost/Hour & vCPUs & Request/second & $\begin{array}{c}\text { 100 Request } \\
\text { Cost/Second }\end{array}$ \\
\hline Small & $\$ 0.15$ & 4 & $92(4 \mathrm{vCPUs} \times 23$ requests/s $)$ & $\$ 0.163$ \\
\hline Medium & $\$ 0.25$ & 8 & $192(8 \mathrm{vCPUs} \times 24$ requests/s $)$ & $\$ 0.130$ \\
\hline Large & $\$ 0.50$ & 16 & $320(16 \mathrm{vCPUs} \times 20$ requests/s $)$ & $\$ 0.156$ \\
\hline
\end{tabular}

Generally, the non-algorithmic approach uses a simple, fixed formula to predict future conditions. the formula consists of several components that affect the result of forecasting. For example, study of sales forecasting in Chinese Tobacco Wholesalers [6] done using a simple formula of population, multiplied with consumer rate, multiplied by consumption rate per day for a person in a certain area. Second approach called by algorithmic approach. This approach uses an advanced technique which enables the forecasting process to adapt along with changes in input data [7], compare several Artificial IntelligenceBased algorithm to predict sales based on historical data, and online reviews. Naive Bayes, Support Vector Machine and K-Nearest Neighbour (KNN) were compared in those studies, they found naive Bayes algorithm excel, especially in sentiment analysis. Indeed, a non-algorithmic approach more convenient to use, yet easily digest and explained. But, the capabilities of the algorithmic approach to adapt with input data give more advantages in long term perspective. With capability to adopt, forecasting system have a better handling of noise, outliers, or even, shifting pattern.

One aspect which affecting sales forecasting is promotion schedule [8]. Promotion, which commonly occurred in form of discount proven effective to boost purchase quantity. It is reported, $20 \%$ of price cut bring $73 \%$ rise in sales $[9,10]$. Since using promotion is commonly done nowadays, especially in internet business, it is hardly seen a day without promotion in Indonesian ecommerce.

Thus, this study proposes a set of steps to forecast transactions a day based on promotion scenario data with main objective is to discover the best machine learning algorithm and model to forecast future sales. Promotion mechanism which employed in this study are price cut, buy 1 -get 1 , and product bundling. We will use dataset from one of e-retail groceries in Indonesia within January 2018 to July 2019.

\section{LITERATURE REVIEW}

Previous study about sales forecasting does not have to use sophisticated algorithm to produce acceptable accuracy. For example [6] simply formulates market demand for tobacco in china as a multiplication of population, consumer rate, and average individual daily consumption. This formula successfully predicts annual sales with an accuracy of $99.18 \%$, while in the monthly forecast, the error rate is variate between $-0.03 \%$ and $7.82 \%$. [11] tried to predict future sales based on browsing history, and individual intention in an electronic commerce site. The study involved 2 observed variables: 1) Browsing ratio; defined as, probability of a customer to visit a page. 2) Browsing Duration; defined as, last visiting page for window-shopper type customer. Proposed model of the study produces higher result of average RMSE compared to CTREND and PTREND methods.

Specific study about sales forecasting, under promotion condition also already done previously, but from the best of our knowledge very limited. [12] investigate online review and online promotional strategies such as free delivery and price cut discount combined with sentiment from user reviews. [13] studied sales prediction in large retail distribution. The study uses SVM as forecasting model and compare the performance to exponential smoothing model (ES), and Holt-Winter triple Exponential Smoothing (HWES). They use MSE as performance metrics to be compared. Uniqueness of the study is they use two categories of attributes: calendar attribute and problem specific attribute. Calendar attribute employed in the study due consideration of typical human behaviour and custom. In the study also, they compared SVM with 2 different number of inputs: 12 and 4. SVM with 4 input successfully outperform another algorithm.

Another study which inspired our study is [14], the study compares existence of promotion factor in sales forecasting. 


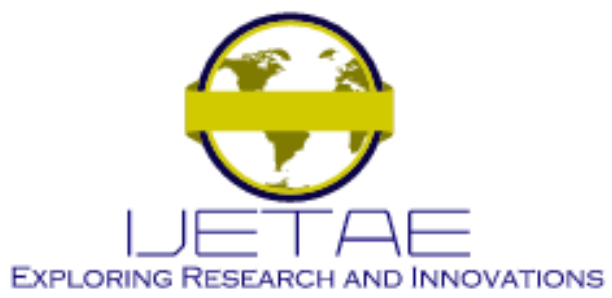

International Journal of Emerging Technology and Advanced Engineering

Website: www.ijetae.com (E-ISSN 2250-2459, Scopus Indexed, ISO 9001:2008 Certified Journal, Volume 11, Issue 06, June 2021)

They use several models which are: SVR, stepwise linear regression, and regression tree. One interesting discussion of the study is, they map the models, into data preparation cost versus technique complexity analysis. At the analysis without promotion, interestingly, none of machine learning algorithm improve the performance of forecasting. With promotion scenario, the study shown regression tree with full features yielded highest result compared to another models. But the result is worse than forecast in no promotion condition with traditional statistics.

From previous study above, general step to done sales forecasting can be taken as an inspiration to this study. First step to be taken in this study is data extraction, the next step is data preparation, then followed by model building. but as we can see in literature review, each model has different parameter and behaviour which affect the prediction accuracy. This difference, affected by variety and amount of the data itself. So, this study will explore the best option for each selected model before we continue to the next step. In terms of model selection, as mentioned in previous study Support Vector Regression/Machine always produces the best accuracy.

\section{A. Generalized Linear Model}

Generalized Linear Models (GLMs) basically is linear combination of rule-based features. This model, naturally interpretable yet relatively complex and flexible, since the non-linearity were able to be captured by rules [15].

Generally, in model fitting process, according to [16] GLM have three processes of model fitting: 1) model selection 2) parameter estimation 3) prediction of future value. These processes also similar with [17] in timeseries analysis. Since current study will be done in RapidMiner Studio, GLM problem consist of three main components, which are:

1) A linear model systematic component $\eta$ : $\eta=X \beta$, where $\mathrm{X}$ is the matrix of all observation vectors $\mathrm{xi}$.

2) The association function expressed by $g$ : $E(y)=\mu=g-1(\eta)$ intent of this component is to link the expected value of $\mu$ to the result of linear component $\eta$. This relaxes the constraints alongside the additivity of the covariates, and it allows the response belonging to a restricted range of values depending on the selected transformation $\mathrm{g}$.
3) The density function $f$ which consists of dependent variable y and parameterized with $\theta$ and $\phi$ which has a probability distribution. This component intended to get rid of the restriction on the distribution of the error and allows for non-homogeneity of the variance with reference to the mean vector. Thus, this component formed as a random $f$ for the variable $y$.

The combination of family function and link function can be formed like well-known algorithm, such as Linear Regression, which basically consist of gaussian family function and identity link function.

\section{B. Support Vector Regression}

Support Vector (SV) method was developed by Vapnik in 1995, initially introduced as Support Vector Machine (SVM) to solve the overlapped classification problem. Instead of minimizing the training error, SV seeks to minimize the upper bound of generalization error. SVM Model returned an output from a finite set. If the model returns a continuous value, the problem solved a regression problem, called by SVR (Support Vector Regression). SVR introduce $\varepsilon$-tub, an $\varepsilon$-insensitive region, around the function. Compared with other neural network models, SV has better generalization performance [18].

\section{K-NN Regression}

In the general classification problem, let $T=$ $\left\{x_{n} \in R^{d}\right\}_{n=1}^{N}$ denote a training set with $\mathrm{M}$ classes consisting of $\mathrm{N}$ training samples in d-dimensional feature space, and the class label of one sample $x_{n}$ is $c_{n}$. Given a query point $x$, the KNN rule is carried out as follows [20].

Let $T=\left\{\left(x_{i}^{N N}, y_{i}^{N N}\right)\right\}_{i=1}^{k}$ indicate the set of $\mathrm{k}$ nearest neighbors for $x$. To find $k$ nearest neighbors from the set $\mathrm{T}$ for the unknown query point $x$, the distance between $x$ and the neighbor $x_{i}^{N N}$ is measured by the distance calculation method, commonly it used Euclidean distance metric.

The class label of the query point $x$ is predicted by the majority voting of its neighbors, expressed by (1)

$$
y^{\prime}=\max \sum_{\left(x_{i}^{N N}, y_{i}^{N N}\right) \in \bar{T}} \delta\left(y=y_{i}^{N N}\right)
$$

where $y^{\prime}$ is a class label and $y_{i}^{N N}$ denotes the class label for the $i^{\text {th }}$ nearest neighbor among its $k$ nearest neighbors. $\delta\left(y=y_{i}^{N N}\right)$, an indicator function takes a value of one if the class $y_{i}^{N N}$ of the neighbor $x_{i}^{N N}$ is the same as the class $y_{i}^{N N}$ and zero otherwise. 


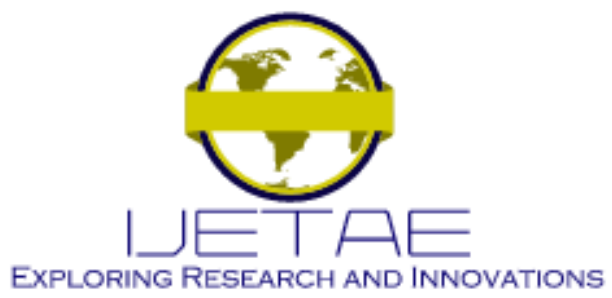

International Journal of Emerging Technology and Advanced Engineering

Website: www.ijetae.com (E-ISSN 2250-2459, Scopus Indexed, ISO 9001:2008 Certified Journal, Volume 11, Issue 06, June 2021)

Nearest neighbor first used as classification given unlabelled data, sample data with the most similar label and class used to predict class from unlabelled data samples. This algorithm is included in the classical algorithm in machine learning [21]. $k$ is an integer and usually, $k$ is greater than unity to avoid overfitting caused by noise. Instances are described by the feature vector and the area between the instances is typically implemented. In this way, the closest example to an unlabelled example, according to the vector feature and the distance function, is the nearest neighbor used to classify the unlabelled example. Nearest neighbor algorithms can easily be extended to regression [22]. With examples where the target value is unknown, the target values of the nearest neighbor are combined, for example with the mean or median, to predict the unknown target value. In this case, the example has an associated numeric target value, not a class or category.

\section{METHODS}

\section{A. Data Extraction}

There are two data which will be extracted, first one is user analytic data, extracted from analytic tools platform. this data contains several metrics such as website and apps views, sessions, number of users, number of transactions, and approximate revenue in million. These data are sum of each in a day. 577 data were extracted from Analytic platform to analysed. The data was extracted in CSV format, but for convenience to merge the data with another sources, it converted into PostgreSQL table.

The second data extracted directly from OLTP storage of the company, which contain promotion data with range time of January 2018 - June 2019. Since huge amounts of data are extracted, data will be remaining in SQL format and put it into single PostgreSQL table, to ease transformation and query process. Attributes which will be used in this study are discount amount, discount percentage, minimum transaction purchased, minimum qty purchased, maximum discount amount, maximum qty applied, maximum promo used per user, maximum promo used, start promo date and time, end promo date and time, promo type, voucher type. 36385 data were extracted from OLTP storage to analysed.
We Also generate a single RDBMS table called 'Master day' which consist of single column, provided running date value from $1^{\text {st }}$ January 2018 to $31^{\text {st }}$ June 2019 . Once all data already collected 3 separated table in single PostgreSQL database, we join all the data, and extracted into single CSV file. This file, furthermore, will be used as source to the next processing step.

\section{B. Data Preparation}

Incomplete, inconsistent, and dirty data can be handled automatically by the system. But in this study, we will not be handling those data by system, thus, manually picking will be done. Since we extract the data directly from production OLTP, First, we will remove unrelated data. Due there is the possibility of testing data, which does not represent actual features in real life. Second, we label the data, according to the company business rule itself, creating a separate dataset for each business rule.

We divide OLTP data into two categories based on promo type: Shopping cart promo and non-shopping cart promo. This division due to significant difference in formed data. Shopping cart-type promo commonly have simpler rule such as fixed amount discount and minimum amount purchased. Thus, these data will not be nulled.

After categorizing based on promo type, extracted data will be combined, promo in a day will be summarized, and new dataset is produced with new attributes. With these combined data, if null value still exists, it means, the promo does not have any limit number, so it will be replaced with highest max discount value in a day. 17 attributes were generated from this step. We also add 5 calendar attributes, which are: day of month, day of week, month in number, is public holiday, and is fasting month. These new attributes added considering behaviour of Indonesia consumer. For example: sales are rising in every $25^{\text {th }}$ day $-1^{\text {st }}$ day of next month due monthly wages. All attribute which generated from this process can be seen in Error! Reference source not found. 


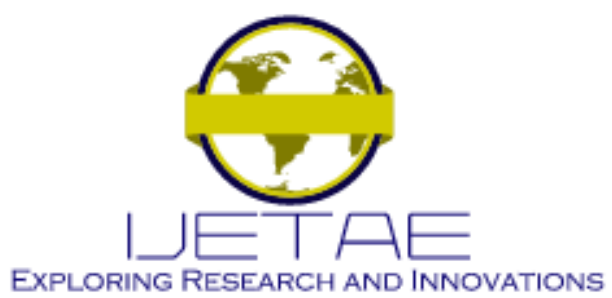

International Journal of Emerging Technology and Advanced Engineering

Website: www.ijetae.com (E-ISSN 2250-2459, Scopus Indexed, ISO 9001:2008 Certified Journal, Volume 11, Issue 06, June 2021)

TABLE 2

SUMMARIZE OF GENERATED ATTRIBUTES

\begin{tabular}{lll}
\hline No & Attributes & Type \\
\hline $\mathbf{1}$ & DayOfMonth & Integer \\
\hline $\mathbf{2}$ & DayOfWeek & Integer \\
\hline $\mathbf{3}$ & Month Number & Integer \\
\hline $\mathbf{4}$ & is Fasting Month & 1 or 0 \\
\hline $\mathbf{5}$ & is public holiday & 1 or 0 \\
\hline $\mathbf{6}$ & Count of promo bundle & integer \\
\hline $\mathbf{7}$ & Count of promo shopping cart & integer \\
\hline $\mathbf{8}$ & number of transactions & integer \\
\hline $\mathbf{9}$ & Count of promo buylget1 & integer \\
\hline $\mathbf{1 0}$ & Count of promo special price & integer \\
\hline $\mathbf{1 1}$ & min max discount amount & double \\
\hline $\mathbf{1 2}$ & avg max discount amount & double \\
\hline $\mathbf{1 3}$ & max max discount amount & double \\
\hline $\mathbf{1 4}$ & max nilai minimum pembelian & double \\
\hline $\mathbf{1 5}$ & min nilai minimum pembelian & double \\
\hline $\mathbf{1 6}$ & discount percentage promo max & double \\
\hline $\mathbf{1 7}$ & discount percentage promo avg & double \\
\hline $\mathbf{1 8}$ & discount percentage promo min & double \\
\hline $\mathbf{1 9}$ & sum of price cut with voucher & integer \\
\hline $\mathbf{2 0}$ & sum of price cut without voucher & integer \\
\hline $\mathbf{2 1}$ & sum of (\%) price cut with voucher & integer \\
\hline $\mathbf{2 2}$ & sum of (\%) price cut without & integer \\
\hline & voucher & \\
\hline
\end{tabular}

Since we have huge different in attribute unit, normalization is needed. Range normalization was done with $\min _{\text {point }}=0$ and $\max _{\text {point }}=1.0$. Range normalization calculated using (2):

$$
\text { normalized }=\frac{\text { raw }_{\text {value }}-\min _{\text {value }}}{\text { max }_{\text {value }}-\min _{\text {value }}}\left(\max _{\text {point }}-\min _{\text {point }}\right)+\min _{\text {point }}
$$

\section{Best Parameter for GLM: Families and Solver}

Since there are some parameters to filled in, in order to solve a GLM problem, a family function (f), and link function (g) were needed to be determined. There are several family options to be examined are: gaussian, binomial, fractional binomial, ordinal, quasibinomial, multinomial, Poisson, gamma, tweedie, and negative binomial.

There are 4 type of solver which will be examined: Gradient Descent Likelihood, and Gradient Descent Squared Error, Coordinate Descent, Coordinate Descent naïve, and Iteratively Reweighted Least Squares Method (IRLSM), Limited memory Broyden-Fletcher-GoldfarbShanno (L_BFGS).

\section{Best Parameter for SVR: C and kernel Type}

Support vector implementation of current study will use implementation of [19], which have two parameter options: parameter set and kernel definition set. In these options, only two variables will be role as independent variable: kernel definition and number of $\mathrm{C}$.

To multiple $\mathrm{C}$ value, we use a minimum of 1.0 and a maximum of 1000 . We use the quadratic scale with 100 steps to generate $\mathrm{C}$ value. We examine 6 kernel type:

- Dot: kernel defined by $k(x, y)=x \times y$,

- Radial: kernel defined by $\exp \left(-\gamma\|x-y\|^{2}\right)$,

- Polynomial: defined as $(x \times y+1)^{d}$,

- Neural: defined by two-layer neural net $\tanh (a x \times y+$ b),

- ANOVA: defined as $\sum \exp \left(-\gamma\|x-y\|^{2}\right)^{d}$,

- Epachnenikov: defined as $\frac{3}{4} \times(1-2 u)$.

\section{E. Best Parameter for KNN: $k$-value and distance calculation method}

$k$ is a user-defined neighbor parameter in the K-NN algorithm, this parameter is used to classify sample to be labelled based on the highest frequency of occurrence among the $\mathrm{k}$ training samples closest to the selected data point. Generally, the value for $\mathrm{k}$ is often specified as a relatively small value but must be an integer. If the k-value is too large or too small, it will increase interference to the data and reduce classification accuracy. In cases where the $\mathrm{k}$ value is small, the complexity of the model is higher (i.e., it is easy to have over-fitting problems) which causes the forecast results to be very sensitive to neighboring points. Conversely, if the value of $\mathrm{k}$ is large, it will reduce the estimation error; However, the forecast error will increase as well, and the training data points that are farther from the training data points will affect the forecast results. In this study grid search optimization method employed to determine the suitable value of $\mathrm{k}$.

In the k-NN classifier, the distance between the test sample and the training sample can be identified by various measurements. Hence, distance measurement plays an important role in determining the final output [23]. Euclidean distance is the most widely used distance measurement in the $\mathrm{k}-\mathrm{NN}$ model, however, only a few studies have examined the effect of different distance measurements on k-NN performance. Either using a small distance or a small number of data sets, or both. 


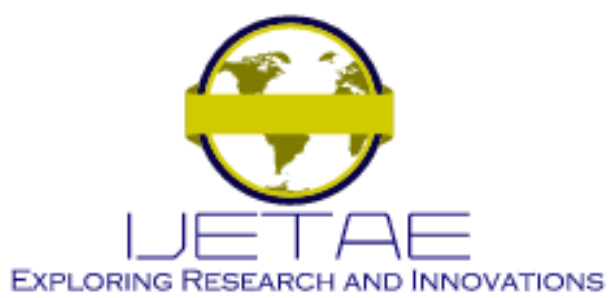

International Journal of Emerging Technology and Advanced Engineering

Website: www.ijetae.com (E-ISSN 2250-2459, Scopus Indexed, ISO 9001:2008 Certified Journal, Volume 11, Issue 06, June 2021)

No experiment has been able to produce the best distance in every k-NN problem. Therefore, in this study grid search optimization method employed to determine the distance calculation method which yielded the best k-NN performance.

\section{F. Experiment design}

This study will use k-NN as predictor model to determine the number of transactions based on promotion scenario data which generated in data pre-processing step. As a benchmark, we will use Support Vector Regression (SVR) and Generalized Linear Model (GLM) to compare performance of k-NN model to predict the value.

Experiment will be done in three steps: training, validation, and testing. We will use 577 data which cover date from January 2018 to July 2019. Data will be shuffled and split by 60:20:20 for each step, respectively. This study will use RapidMiner Studio (C) version 9.8.0.

\section{G. Performance evaluation}

For evaluate performance of the model, we use 3 performance metrics, which are: correlation (R), squared correlation $\left(\mathrm{R}^{2}\right)$, and Mean Absolute Error (MAE).

Correlation, can be calculated by (3):

$$
R=\frac{n \times \sum x_{i} y_{i}-\sum x_{i} \sum y_{i}}{\sqrt{n \times \sum x_{i}^{2}-\left(\sum x_{i}\right)^{2}} \times \sqrt{n \times \sum y_{i}^{2}-\left(\sum y_{i}\right)^{2}}}
$$

Squared correlation can be calculated using (4):

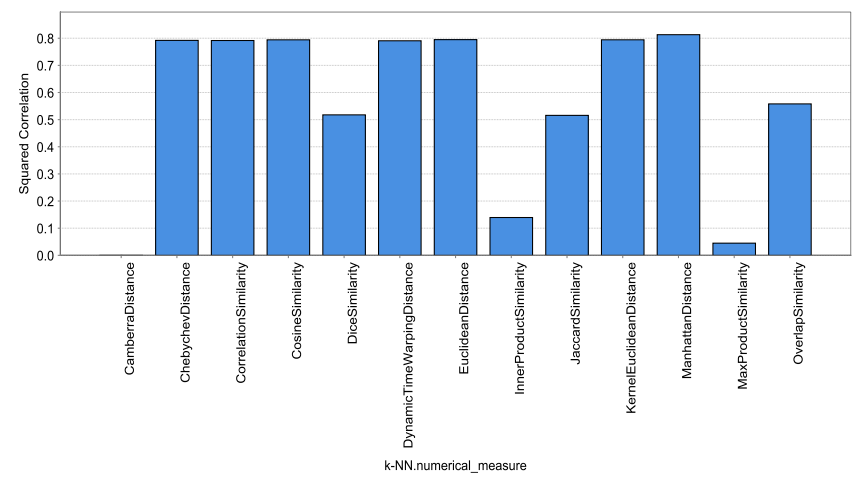

(a)

$$
R^{2}=1-\frac{S S_{r e s}}{S S_{t o t}}
$$

$\mathrm{SS}_{\text {tot }}$ and $\mathrm{SS}_{\text {res }}$ expressed by (5) and (6) respectively:

$$
\begin{aligned}
& S S_{\text {tot }}=\sum\left(x_{i}-\bar{x}\right)^{2} \\
& S S_{\text {res }}=\sum\left(x_{i}-y_{i}\right)^{2}
\end{aligned}
$$

Mean absolute error calculated using (7):

$$
M A E=\frac{\sum\left|y_{i}-x_{i}\right|}{n}
$$

Where,

$\bar{x}=$ average of training value

$\mathrm{n}=$ number of data

$\mathrm{x}_{\mathrm{i}}=$ training value

$\mathrm{y}_{\mathrm{i}}=$ predicted value

\section{RESUlt AND Discussion}

We determine the best distance calculation method which yielded the highest performance of k-NN. We examine 13 distance calculation method. To define the best distance calculation method, we compare squared correlation (R2) of each k-NN output with examined distance calculation, then, distance calculation which yield the highest metrics will be selected. From the experiment, as seen in

Figure 1a, although the performance result similar with other 6 calculation method, we discover Manhattan distance yield the highest R2 of 0.813 .

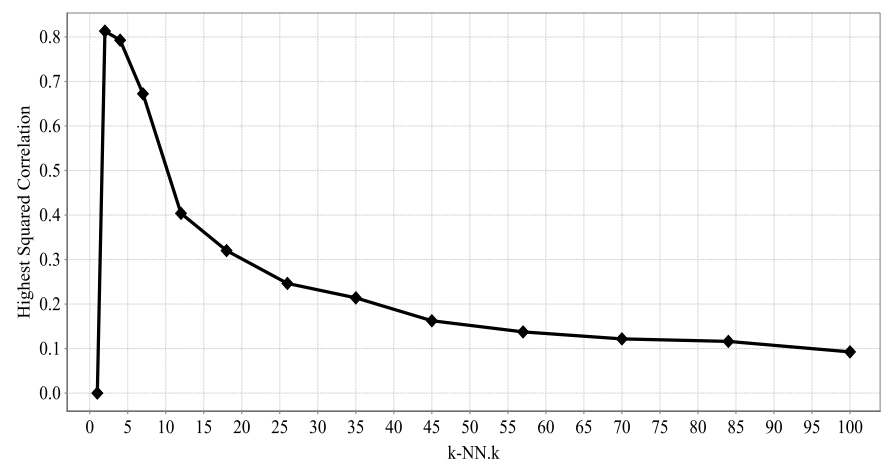

(b)

Figure 1 a) Comparison of distance calculation method b) Comparison of k-value to k-NN performance 


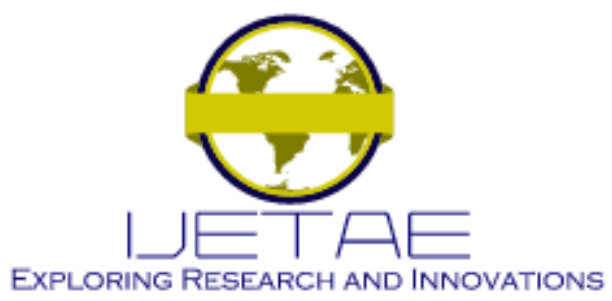

International Journal of Emerging Technology and Advanced Engineering Website: www.ijetae.com (E-ISSN 2250-2459, Scopus Indexed, ISO 9001:2008 Certified Journal, Volume 11, Issue 06, June 2021)

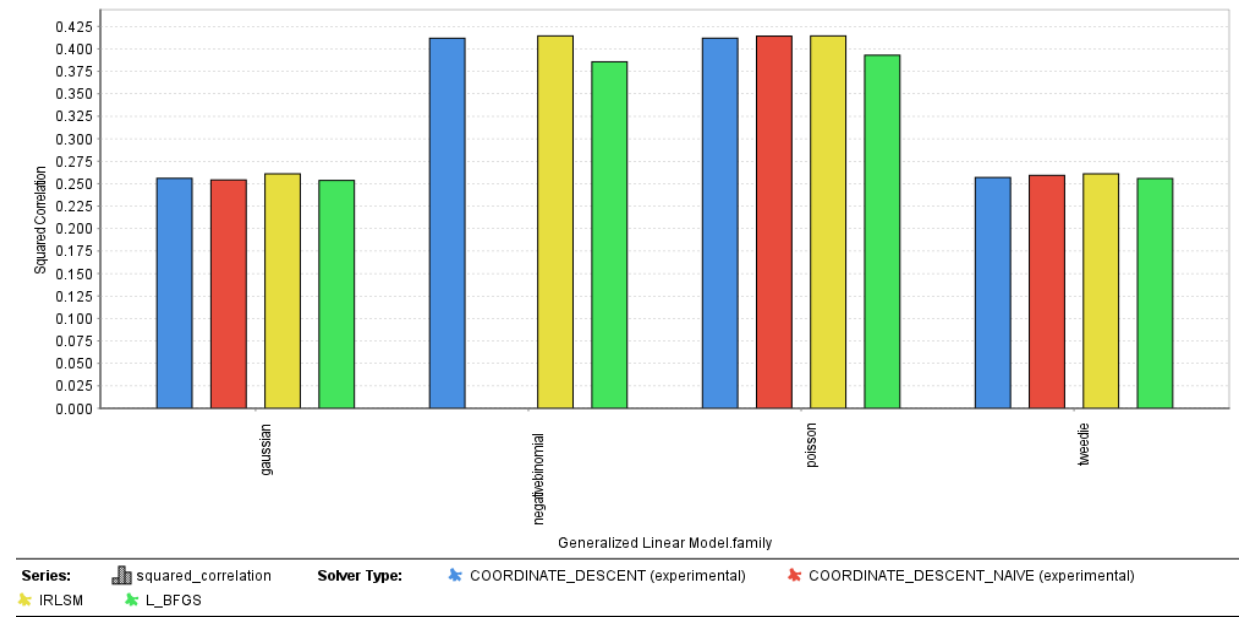

Figure 2 GLM parameter comparison

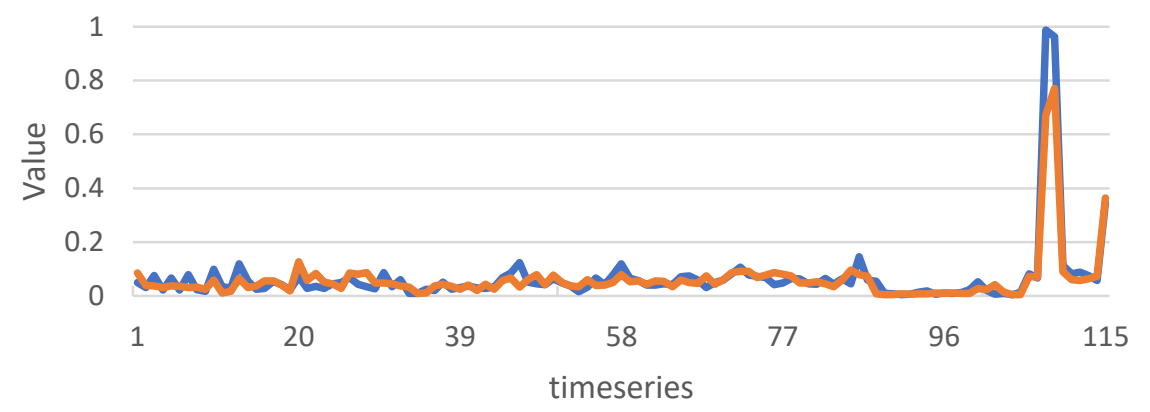

transaction $\longrightarrow$ prediction

Figure 3 Visualization result of $k-\mathrm{NN}$ prediction

From Figure $1 \mathrm{~b}$, we determine $\mathrm{k}$ value for $k-N N$ parameter, in this study, we use minimum value of 1 and maximum value of 100 with 12 steps and using quadratic scale. Thus, there are $13 k$ number which we examine, that are: $1,2,4,7,12,18,26,35,45,57,70,84,100$. Searching for best $\mathrm{k}$ value done in validation set data. For each experiment, we compare squared correlation $\left(\mathrm{R}^{2}\right)$ metrics for each k-NN output, then, $\mathrm{k}$ value which yield the highest metrics will be selected. From the experiment we discover the best $\mathrm{k}$ value is 2 , which yield $\mathrm{R}^{2}$ of 0.813 .

For GLM model to determine the best parameter, we examine family and solver parameter of the model with squared correlation as performance metrics. As shown at Figure 2, 4 family examined which are: negative binomial, tweedie, Poisson, and gaussian.
And for solver parameter we examined 4 of them which are: IRLSM, Coordinate Descent, L_BFGS, and Coordinate Descent Naïve. Gaussian family have relatively equal in squared correlation for all solver involved, this result also occurred on tweedie family, which have relatively equal for squared correlation for all solvers. Poisson and negative binomial on the other hand, yield the highest squared correlation compared to another two families. Negative binomials yield the best result while combined with Coordinate Descent and IRLSM, while combined with Coordinate Descent Naïve, the result was completely zero. We concluded the best parameter for family is Poisson and the best solver parameter is Coordinate Descent Naïve. 


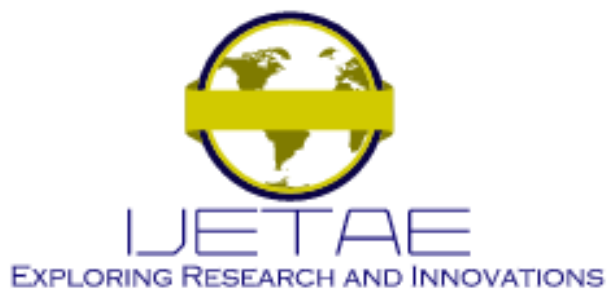

International Journal of Emerging Technology and Advanced Engineering

Website: www.ijetae.com (E-ISSN 2250-2459, Scopus Indexed, ISO 9001:2008 Certified Journal, Volume 11, Issue 06, June 2021)

In SVR, we run experiment of 6 kernel type: dot, radial, polynomial, neural, ANOVA and epachnenikov as shown in Figure 4. Radial kernels yield the highest result with average squared correlation of 0.765 , while dot kernel result the lowest result with 0.002 . Anova, epachenikov, neural, and polynomial kernels yield result of $0.569,0.641$, 0.03 , 0.066, respectively. For complexity value $(\mathrm{C})$, we examine value with minimum of 1.0 and maximum of 1000. We use quadratic scale with 100 steps. We measured maximum squared correlation produced for each Complexity value. From this experiment we discovered that the highest result yielded at $\mathrm{C}$ value of 1 , and it decreased dramatically until the lowest result at $\mathrm{C}$ value of 3.498 , and the squared correlation raise again at $\mathrm{C}$ value of 4.596, after this point the result are equals, as shown in Figure 5. We concluded the best parameter value of SVR kernel is radial and the best complexity value is 1 .

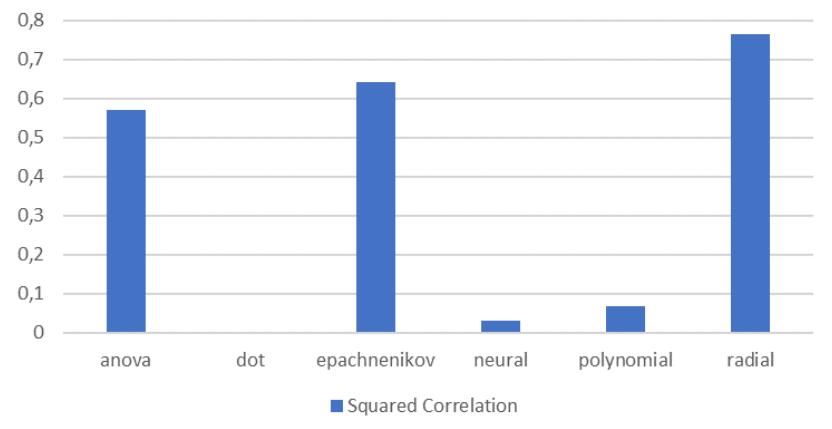

Figure 4 SVR kernel comparison

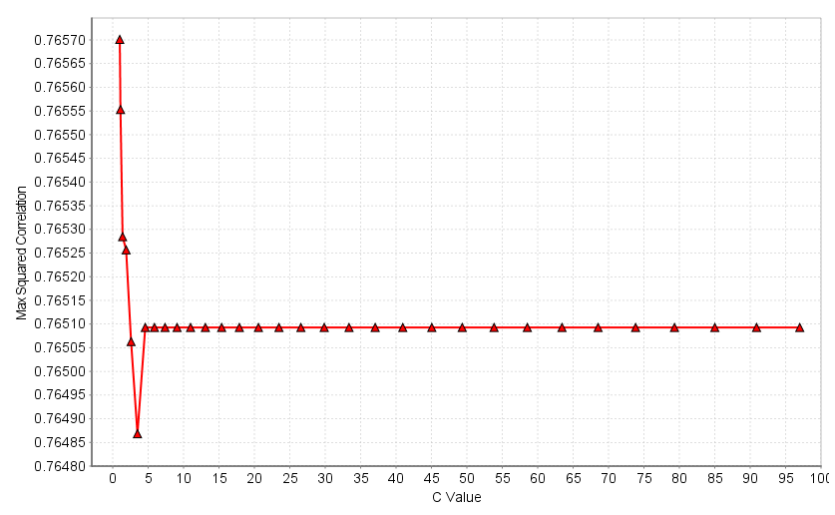

Figure 5 SVR Complexity value comparison
Performance comparison of k-NN, GLM and SVR model can be seen in Table 1. k-NN yield the highest squared correlation with 0.938 , followed by SVR with 0.891 and last one is GLM with 0.507. Mean absolute error also show the similar behaviour with squared correlation, as we can see k-NN resulted the lowest MAE with 0.022 , followed by SVR with 0.029, and the highest MAE produced by GLM with 0.041 .

Table 1:

Performance comparison for models

\begin{tabular}{|l|c|c|c|}
\hline \multirow{2}{*}{ Model } & \multicolumn{3}{|c|}{ Performance Metrics } \\
\cline { 2 - 4 } & MAE & $\mathrm{R}$ & $\mathrm{R}^{2}$ \\
\hline KNN & $0.022+/-0.036$ & 0.969 & 0.938 \\
\hline SVR & $0.029+/-0.056$ & 0.944 & 0.891 \\
\hline GLM & $0.041+/-0.088$ & 0.712 & 0.507 \\
\hline
\end{tabular}

Another interesting analysis we could gather from SVR and GLM algorithm is importance of each attribute using coefficient produced by the output model. Attribute which has high significance for calculation have coefficient greater than 0 , and less than zero. Greater or lesser the coefficient number, greater impact given by the attribute to the calculation result. Negative coefficient means it has inverse contribution for the calculation result, while positive coefficient means the attribute give directly positive proportional contribution for the calculation result.

Figure 6 shown, coefficient of each attribute produced by GLM algorithm. From the graph, we can see the attributes coefficient lays from -5 to 3 . The most significance attribute for the calculation result have a coefficient value near to -5 or 3 . On the other side, the most insignificance attribute for the calculation result have a coefficient value near 0 . The most insignificance attribute that easily recognized is fixed discount promo with nonvoucher mechanism with coefficient of 0.03 . The most positively significance attribute is average of discount percentage promo in a day with coefficient of 3.072. And for the most negative impact attribute is count of direct promo price discount in a day with coefficient of -4.131 . 


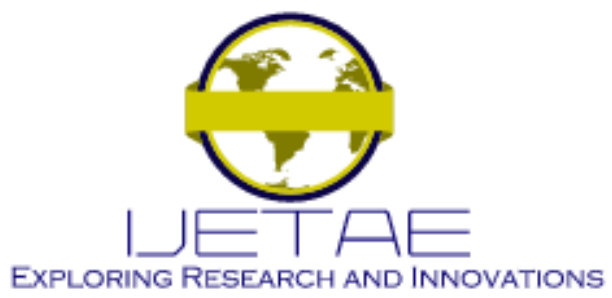

International Journal of Emerging Technology and Advanced Engineering Website: www.ijetae.com (E-ISSN 2250-2459, Scopus Indexed, ISO 9001:2008 Certified Journal, Volume 11, Issue 06, June 2021)

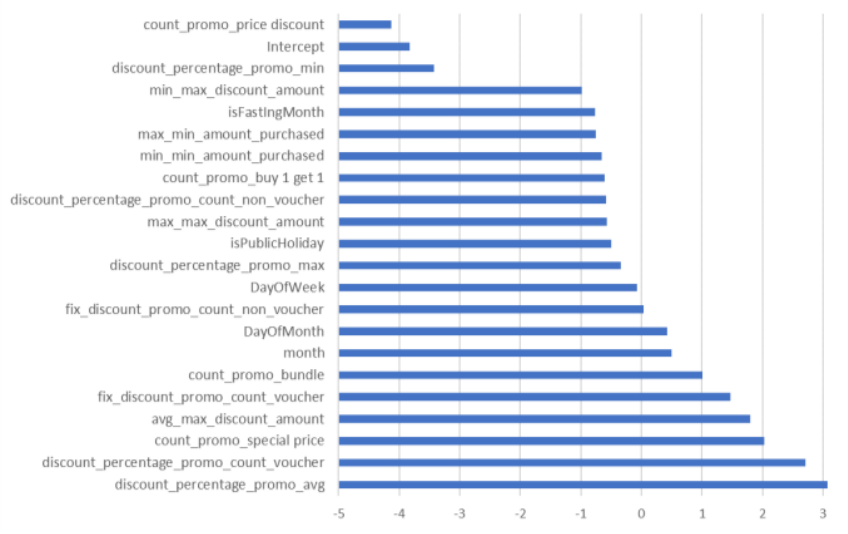

Figure 6 Coefficient of each attribute from GLM algorithm

Attribute coefficient produced by SVR algorithm can be seen in Figure 7. From the graph, we can see the coefficient attribute lays from -3 to 5 . Generally, all attributes have significance contribution to calculation result, which make the analysis of most insignificance quite hard, but the nearest value to 0 are owned by count of promo price discount which have coefficient of 0.415 , while the most positively significance attribute goes to attribute day of month with coefficient of 5.203 and the most negative impact attribute goes to attribute minimum value of minimum amount purchased in a day with coefficient of 2.380 .

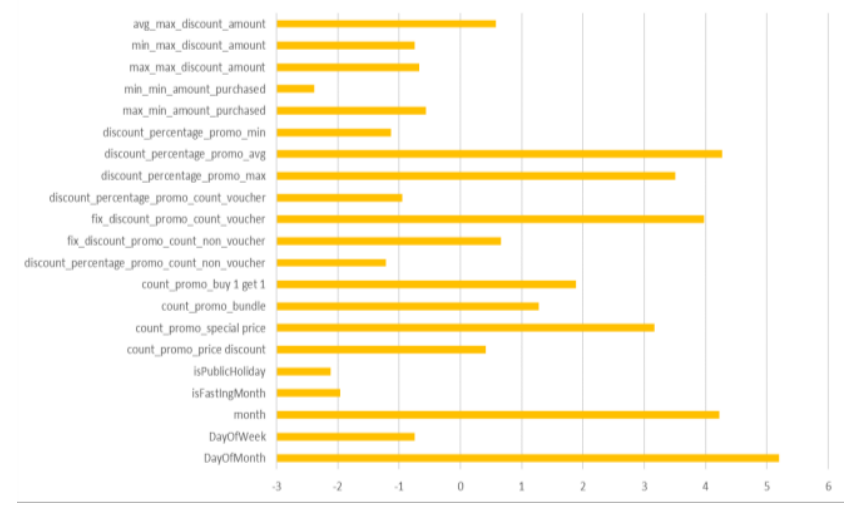

Figure 7 Coefficient of each attribute from SVR algorithm

\section{CONCLUSION}

This study aimed to predict number of transactions a day using promotion data using one of Indonesia e-retail groceries. We use 577 data from January 2018 to July 2019 as dataset. We compare k-NN, GLM, and SVR as the model predictor to forecast number of transactions in a day. From the experiment k-NN yielded the highest performance ability with squared correlation of 0.938 . the worst model predictor for this case is GLM with squared correlation of 0.507 . We also determine the best parameter input for each parameter using grid optimization method. We discover the best $\mathrm{k}$ value is 2 and Manhattan distance is the best distance calculation for this case.

From this study, we also discover that sales forecasting problem seen as simple regression problem, instead needed to be tackled with complicated timeseries analysis. But future exploration can be made to determine whether using timeseries analysis could boost the performance of the prediction.

\section{REFERENCES}

[1] K. Goetsch, eCommerce in the Cloud, O'Reilly Media, Inc, Sebastopol, 2014.

[2] P. Doganis, A. Alexandridis, P. Patrinos, H. Sarimveis, "Time series sales forecasting for short shelf-life food products based on artificial neural networks and evolutionary computing," Journal of Food Engineering, $\quad \mathbf{7 5}(2), \quad 196-204, \quad 2006$, doi:10.1016/j.jfoodeng.2005.03.056.

[3] K. Chen, "Combining linear and nonlinear model in forecasting tourism demand," Expert Systems With Applications, 38(8), 10368 10376, 2011, doi:10.1016/j.eswa.2011.02.049.

[4] G. Kulkarni, P.K. Kannan, W. Moe, "Using online search data to forecast new product sales," Decision Support Systems, 52(3), 604611, 2012, doi:10.1016/j.dss.2011.10.017.

[5] F. Martínez, M.P. Frías, M.D. Pérez-Godoy, A.J. Rivera, "Dealing with seasonality by narrowing the training set in time series forecasting with kNN," Expert Systems with Applications, 103, 3848, 2018, doi:10.1016/j.eswa.2018.03.005.

[6] D. Fanga, W. Weng, "Procedia Environmental Sciences Sales Forecasting System for Chinese Tobacco Wholesalers," Procedia Environmental Sciences, 11, 380-386, 2011, doi:10.1016/j.proenv.2011.12.061.

[7] Z. Fan, Y. Che, Z. Chen, "Product sales forecasting using online reviews and historical sales data: A method combining the Bass model and sentiment analysis," Journal of Business Research, 74 90-100, 2017, doi:10.1016/j.jbusres.2017.01.010. 


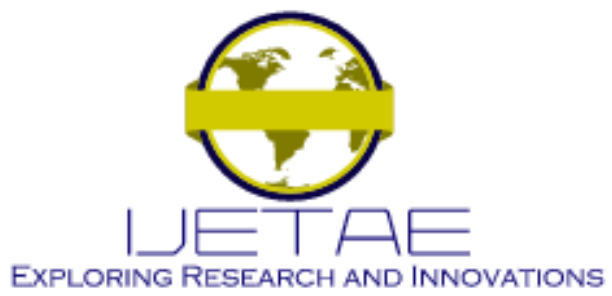

International Journal of Emerging Technology and Advanced Engineering Website: www.ijetae.com (E-ISSN 2250-2459, Scopus Indexed, ISO 9001:2008 Certified Journal, Volume 11, Issue 06, June 2021)

[8] S. Ma, R. Fildes, T. Huang, "Demand forecasting with high dimensional data: the case of SKU retail sales forecasting with intraand inter-category promotional information," European Journal of Operational Research, 2015, doi:10.1016/j.ejor.2015.08.029.

[9] M.G. Dekimpe, D.M. Hanssens, V.R. Nijs, J.B.E.M. Steenkamp, "Measuring short- and long-run promotional effectiveness on scanner data using persistence modelling," Applied Stochastic Models in Business and Industry, 21(4-5), 409-416, 2005, doi:10.1002/asmb.581.

[10] T.H.A. Bijmolt, H.J. Van Heerde, R.G.M. Pieters, "New empirical generalizations on the determinants of price elasticity," Journal of Marketing Research, 42(2), 141-156, 2005, doi:10.1509/jmkr.42.2.141.62296.

[11] J. Yeo, S. Kim, E. Koh, S. Hwang, N. Lipka, "Browsing2purchase : Online Customer Model for Sales Forecasting in an E-Commerce Site," in Proceedings of the 25th International Conference on World Wide Web, 133-134, 2016.

[12] Alain Chong Boying Li EWT Ngai Eugene Ch'ng Filbert Lee, Predicting online product sales via online reviews, sentiments, and promotion strategies: a big data architecture and neural network approach, 2016.

[13] G. Di Pillo, V. Latorre, S. Lucidi, E. Procacci, "An application of support vector machines to sales forecasting under promotions," 4or, 14(3), 309-325, 2016, doi:10.1007/s10288-016-0316-0.

[14] Ö.G. Ali, S. Sayin, T. van Woensel, J. Fransoo, "SKU demand forecasting in the presence of promotions," Expert Systems with Applications, 36(10), 2009, doi:10.1016/j.eswa.2009.04.052.

[15] P. Linardatos, V. Papastefanopoulos, S. Kotsiantis, "Explainable AI: A Review of Machine Learning Interpretability Methods," Entropy, 23(1), 18, 2020, doi:10.3390/e23010018.
[16] P. McCullagh, J.A. Nelder, Generalized Linear Models, 2nd Edn., Applied Statistics, 39(3), 385, 1990, doi:10.2307/2347392.

[17] G.E.P. Box, G.M. Jenkins, G.C. Reinsel, G.M. Ljung, TIME SERIES ANALYSIS : Forecasting and Control, Fifth, John Wiley \& Sons, Inc., New Jersey.

[18] F.E.H. Tay, L.J. Cao, "Application of support vector machines in Financial time series forecasting," Lecture Notes in Computer Science (Including Subseries Lecture Notes in Artificial Intelligence and Lecture Notes in Bioinformatics), 1983, 268-273, 2000, doi:10.1007/3-540-44491-2_38.

[19] T. Joachims, Making large-scale SVM learning practical, 169-184, 1998.

[20] M. Huang, R. Lin, S. Huang, T. Xing, "A novel approach for precipitation forecast via improved K-nearest neighbor algorithm," Advanced Engineering Informatics, 33, 89-95, 2017, doi:10.1016/j.aei.2017.05.003.

[21] X. Wu, V. Kumar, Q.J. Ross, J. Ghosh, Q. Yang, H. Motoda, G.J. McLachlan, A. Ng, B. Liu, P.S. Yu, Z.H. Zhou, M. Steinbach, D.J. Hand, D. Steinberg, Top 10 algorithms in data mining, 2008, doi:10.1007/s10115-007-0114-2.

[22] N.S. Altman, "An introduction to kernel and nearest-neighbor nonparametric regression," American Statistician, 46(3), 175-185, 1992, doi:10.1080/00031305.1992.10475879.

[23] L.Y. Hu, M.W. Huang, S.W. Ke, C.F. Tsai, "The distance function effect on k-nearest neighbor classification for medical datasets," SpringerPlus, 5(1), 2016, doi:10.1186/s40064-016-2941-7. 